\title{
Orientations of Spin Bundles and Symplectic Cobordism
}

\author{
By \\ Vassily GorBunOV* and Nigel RAY**
}

\begin{abstract}
We analyse certain cofibrations of projective spaces in terms of Thom complexes of Spin bundles, and by applying the symplectic cobordism functor we are able to deduce new relations amongst the elements $\dot{\varphi}_{\iota}$ in the symplectic bordism ring.
\end{abstract}

\section{$\S 1$. Introduction}

Symplectic cobordism is the cohomology theory which arises from the Thom spectrum $M S p$ associated with the infinite symplectic group.

For several years now, determining the structure of the coefficient ring $M S p_{*}$ has been seen as an intractible problem, despite the herculean efforts of S. Kochman $[14,15]$ to elucidate the classical mod 2 Adams spectral sequence. Additional recent developments, also often invoking the Adams and Novikov spectral sequences, have tended to be more qualitative, such as the work of V.V. Vershinin and his school $[26,5,10]$ introducing singularities based on certain torsion elements $\phi_{i}$ defined long ago by the second author [20]. Other Soviet workers have made progress by applying the concept of two-valued formal groups, for example see $[6,3]$, but all these studies have involved a high degree of technical complexity, and general interest in the problem has predictably waned.

Lately, however, it has seemed that new developments in related areas might lead to a resurgence of activity. For example, the emergence of elliptic cohomology [16] as an exciting field of contact between algebraic topology, analysis and theoretical physics, suggests that we might look for a real version which is better behaved at the prime 2. By analogy with the relationship between real and complex $K$-theory, this could well arise by imposing certain singularities on symplectic cobordism. Moreover, the remarkable results of $M$.

Communicated by K. Saito, March 5, 1990.

1991 Mathematics Subject Classifications: 55N22, 55R25.

* Mathematics Institute, Siberian Branch of the Soviet Academy of Science, Novosibirsk 630090, USSR.

** Mathematics Department, University of Manchester, Manchester M13 9PL, England. 
Hopkins and his co-workers [12] concerning nilpotency in homotopy rings indicate that we might profitably investigate the special case of $M S p_{*}$ with a view to obtaining more explicit information. And finally, the long standing challenge of axiomatising symplectic orientable cohomology theories, of which symplectic cobordism is the universal example, may be more realistically considered in the context of recent new perspectives [21] on the complex case.

In our view, the study of $M S p$ still stands to gain from the consideration of various phenomena central to real $K$-theory, such as the relationships between the eight Bott periodicity spaces [7], and the assignment by Clifford algebra of $K O$ orientations to arbitrary Spin bundles [2]. It is our aim in this article to support the latter of these claims, by setting up exact sequences incorporating the symplectic cobordism of real, complex and quaternionic projective spaces, and then using $M S p$ orientations of low dimensional Spin bundles to draw conclusions concerning their module structures over $M S p^{*}$. As application, we obtain important new properties of the elements $\phi_{2}$, proving that certain triple products are zero and finding upper bounds for their order of nilpotence. In fact this strategy bears a marked resemblance to that of [20], where a discussion of the modules $M S p_{*}(B S p)$ and $M S p_{*}\left(H P^{\infty}\right)$ led to the definition of the elements $\dot{\phi}_{\imath}$ and the investigation of their elementary properties.

We like to believe that this work gives some hope for the future of symplectic cobordism, in the sense that fairly elementary geometric techniques can still be shown to lead to an infinite amount of new and fascinating information, suggesting that there may yet be more order amidst the chaos of $M S p_{*}$ than meets the eye.

We now give a summary of the contents of each of our sections, noting that $\S \S 2$ and 3 consist mainly of background information and the establishment of notation. We begin in $\S 2$ with the elements $\phi_{\imath}$, explaining V.M. Buhštaber's generalization $\theta_{i}(n)$ in $M S p^{*}\left(R P^{n}\right)$ and describing two special relations in the case $n=2$. In $\S 3$ we discuss low dimensional Spin bundles, paying particular attention to the $M S p$ orientation class of the universal $\operatorname{Spin}(3)$ bundle introduced by N. Ray and R. Switzer in [22]. We outline an alternative approach to this orientation due to K. Kozima and H. Toda [25], and collate material from both sources concerning quaternionic quasi projective space. We are then able to establish useful properties of the cofibre sequences linking real, complex, quaternionic and quasi projective spaces. In passing, we also identify a symplectic analogue of the well-known 2-series in $M U^{2}\left(C P^{\infty}\right)$, which is so influential in the study of $M U$ and $B P$ at the prime 2 [19].

We apply symplectic cobordism to these cofibrations in $\$ 4$, thus obtaining our crucial long exact sequences. With the aid of information on certain symplectic Euler classes $e$ and $d$ culled from $\S 2$, we interpret these long exact sequences in terms of the module structure of $I S p^{*}\left(R P^{\infty}\right)$ and $M S p^{*}\left(C P^{\infty}\right)$, further enhancing details of the latter by proving that any symplectic Euler 
class of a $\operatorname{Spin}(5)$ bundle is zero.

In $\S 5$ we analyse certain symplectic bundles over $R P^{2} \wedge C P^{\infty}$ and $R P^{2} \wedge H P^{\infty}$, and so define new elements $\phi_{2}(n)$ in $M S p^{*}\left(C P^{n}\right)$ which are complex analogues of $\theta_{\imath}(n)$. By incorporating further material from $\S \S 2$ and 3 , we then evaluate the coboundary of the square of the Euler class $d$, and develop explicit formulae relating $\psi_{i}(1)$ and $\theta_{i}(1)$.

These relationships are central to our final $\S 6$, which gives the applications to the structure of $M S p_{*}$. We prove that

$$
\eta \phi_{i} \dot{\phi}_{j}=0 \quad \forall i, j \geqq 1,
$$

where $\eta$ denotes the standard generator of the 1-stem $\pi_{1}^{S}$ and its isomorphic image $M S p_{1}$, thus extending the earlier relation $\eta^{2} \phi_{i}=0$ due to Buhštaber and the first author; an alternative interpretation is that $\eta$ annihilates any symplectic Euler class of a $\operatorname{Spin}(3) \times \operatorname{Spin}(3)$ bundle. As shown by K. Morisugi [17], and independently by Vershinin [27], not all triple products of torsion elements in $M S p_{*}$ are zero.

To conclude, we derive the nilpotency formula

$$
\phi_{i}{ }^{2 l+3}==0 \quad \forall i \geqq 1
$$

from the properties of the elements $\psi_{i}(n)$. This is the first explicit bound known to us for the order of nilpotence of $\phi_{\imath}$ (guaranteed to exist by [12]), although M. Mahowald informs the first author that a simple Novikov spectral sequence argument proves that $\phi_{i}{ }^{82}=0$.

It is a great pleasure for the second author to acknowledge the finanical support provided by both the Royal Society and the Soviet Academy of Science, and to give thanks for the remarkable hospitality shown to him by the Soviet topologists of Novosibirsk; without these, our work could not have been conceived. Both authors also owe a special debt to Boris Botvinnik and Vladimir Vershinin, for many happy hours of discussion and much encouragement.

A preliminary version of part of our work, based on the alternative perspective offered by transfer maps, is given in [11].

\section{$\S 2$. Torsion Elements}

In all that follows, we shall write $\boldsymbol{F}^{n}$ for the trivial $n$-plane vector bundle over any base space (where $\boldsymbol{F}$ denotes $\boldsymbol{R}, \boldsymbol{C}$ or the quaternions $\boldsymbol{H}$ respectively), and $\eta, \zeta$ and $\xi$ for the corresponding Hopf line bundle over $R P^{\infty}, C P^{\infty}$ and $H P^{\infty}$. To avoid over-elaboration, we shall often use the same symbol for a Hopf bundle restricted to different skeleta, and similarly for any vector bundle, its isomorphism class and its classifying map: we trust that the context will always be sufficiently clear to prevent confusion. On occasion, we shall utilise $r, c$ and $q$ to signify the respective operations of realification, complexification 
and symplectification on appropriate bundles or spaces.

We shall let $\bar{\zeta}$ stand for the complex conjugate of $\zeta$, and $\xi^{*}$ for the quaternionic conjugate of $\xi$; since the quaternions do not commute, we must decree in advance that they act on the right of $\xi$, and therefore on the left of $\xi^{*}$. Thus we can form the real 4-plane bundle $\xi \bigotimes_{H} \xi^{*}$, which may easily be identified (for example see [18]) with $\chi \oplus \boldsymbol{R}$, where $\chi$ is the universal $\operatorname{Spin}(3)$ bundle over $B \operatorname{Spin}(3) \simeq H P^{\infty}$.

Recall from [8] that $M S p^{*}\left(H P^{\infty}\right)$ is a free $M S p^{*}$ module; indeed, there is an isomorphism

$$
M S p^{*}\left(H P_{+}^{\infty}\right) \cong M S p_{*}[[z]]
$$

where $z \in M S p^{4}\left(H P^{\infty}\right)$ is our abbreviation for the first Conner-Floyd symplectic Pontrjagin class $p f_{1}(\xi)$. Thus for any space $X$ there is a Künneth isomorphism

$$
M S p^{*}\left(X \wedge H P_{+}^{\infty}\right) \cong M S p^{*}(X)[[z]] .
$$

In similar vein, it will also be helpful to reserve special notation for the suspension isomorphism. Thus we write $s(n)$ for the generator of $M S p^{*}\left(S^{n}\right)$ over $M S p^{*}$, chosen such that it is the exterior product $s(1) \otimes \cdots \otimes s(1)$ under a suitable homeomorphism $S^{n} \doteq S^{1} \wedge \cdots \wedge S^{1}$. Then the suspension isomorphism

$$
M S p^{*+n}\left(S^{n} \wedge Y\right) \cong M S p^{*}\left(Y^{\prime}\right)
$$

will be written as $s(n) y \mapsto y$ for all $y=M S p^{*}(Y)$. Sometimes we shall further abbreviate $s(1)$ to $s$ and $S^{1} \wedge X$ to $S X$.

Now consider the symplectic line bundle $(\eta-\boldsymbol{R}) \otimes_{\boldsymbol{R}}(\boldsymbol{\xi}-\boldsymbol{H})$ over $R P^{n} \wedge H P^{\infty}$. Following Buhštaber, for each $i \geqq 1$ and $1 \leqq n \leqq \infty$ we define $\theta_{\imath}(n)$ in $M S p^{4-4 \imath}\left(R P^{n}\right)$ by applying 2.1 to write

$$
p f_{1}\left((\eta-\boldsymbol{R}) \otimes_{R}(\xi-\boldsymbol{H})\right)=\sum_{\imath \geqq 1} \theta_{i}(n) z^{2}
$$

in $M S p^{4}\left(R P^{n} \wedge H P^{\infty}\right)$. Clearly, given any $m<n$, each $\theta_{\imath}(n)$ restricts to $\theta_{\iota}(m)$ under the inclusion $R P^{m} \subset R P^{n}$.

It follows from the definitions that $\theta_{2}(1)$ in $M S p^{4-4 \imath}\left(R P^{1}\right)$ may be identified with $\theta_{i}$ of [20] in $M S p_{4 i-3}$. We shall thus often find it convenient to relabel $\theta_{1}$ as $\eta$ and $\theta_{2 i}$ as $\phi_{\imath}$, since $\theta_{2 i+1}$ is zero for all $i \geqq 1$ by Roush's theorem [23]. As proved in [20], each $\phi_{i}$ is an indecomposable torsion element of order 2. So we may now rewrite 2.2 in the special case $n=1$ as

$$
p f_{1}\left((\eta-\boldsymbol{R}) \bigotimes_{R}(\xi-\boldsymbol{H})\right)=s\left(\eta z+\sum_{i \geqq 1} \phi_{i} z^{2 \imath}\right)
$$

over $S H P^{\infty}$.

We shall need two simple properties of the elements $\theta_{i}(2)$. For their statement, we choose $r(2)$ in $M S p^{2}\left(R P^{2}\right)$ to be the pull-back $g^{*} s(2)$ along the projection map $g: R P^{2} \rightarrow S^{2}$ onto the top cell. 
Proposition 2.4. The relations

$$
2 \theta_{i}(2)=\eta \theta_{i} r(2) \text { and } \theta_{i}(2) \theta_{\jmath}(2)=\theta_{\imath} \theta_{\jmath} r(2)
$$

hold in $M S p^{*}\left(R P^{2}\right)$ for all $i, j>0$.

Proof. By standard bundle computations (for example see [13]), $g *(r \zeta)=2 \eta$ over $R P^{2}$, and

$$
(\eta-R) \otimes_{R}(\eta-R)=r \zeta-R^{2}
$$

over $S^{1} \wedge S^{1} \doteq S^{2}$. Thus by 2.2

$$
\begin{aligned}
g^{*} p f_{1}\left((\eta-\boldsymbol{R}) \otimes_{R}\left((\eta-\boldsymbol{R}) \otimes_{\boldsymbol{R}}(\boldsymbol{\xi}-\boldsymbol{H})\right)\right) & =p f_{1}\left(2(\eta-\boldsymbol{R}) \otimes_{\boldsymbol{R}}(\boldsymbol{\xi}-\boldsymbol{H})\right) \\
& =2 \sum_{i \geq 1} \theta_{i}(2) z^{2}
\end{aligned}
$$

in $M S p^{*}\left(R P^{2} \wedge H P^{\omega}\right)$. Moreover, by iterating 2.3 and using the fact that $s(1)^{2}$ $=0$ in $M S p^{*}\left(S^{1}\right)$, we obtain

$$
\begin{aligned}
p f_{1}\left((\eta-\boldsymbol{R}) \otimes_{\boldsymbol{R}}\left((\eta-\boldsymbol{R}) \otimes_{R}(\xi-\boldsymbol{H})\right)\right) & =\eta s(1) \otimes s(1)\left(\sum_{\imath \geqq 1} \theta_{i} z^{2}\right) \\
& =\eta s(2) \sum_{i \geqq 1} \theta_{\imath} z^{2}
\end{aligned}
$$

in $M S p^{*}\left(S^{2} \wedge H P^{\infty}\right)$.

Applying $g^{*}$ and equating coefficients of $z^{2}$ now yields the first relation.

For the second, note that the diagonal map $R P^{2} \rightarrow R P^{2} \wedge R P^{2}$ factors as the composition of the projection $g$ with the inclusion of the bottom cell. Thus

$$
\begin{aligned}
\theta_{\imath}(2) \theta_{\jmath}(2)=g^{*}\left(\theta_{i} s(1) \otimes \theta_{\jmath} s(1)\right) & =g^{*}\left(\theta_{i} \theta_{\jmath} s(2)\right) \\
& =\theta_{i} \theta_{j} r(2)
\end{aligned}
$$

as sought.

Corollary 2.5. For all $i, j \geqq 1$, we have that $2 \theta_{\imath}(2) \theta_{\jmath}(2)=0$ in $M S p^{*}\left(R P^{2}\right)$.

\section{$\S 3$. Spin Bundles and Quasi Projective Space}

We now turn our attention to certain important cofibre sequences. They have their origin in the well-known fact (for example see [2]) that, for any $n>1$, the universal $S^{n-1}$ bundle over $\operatorname{BSpin}(n)$ may be written up to homotopy equivalence as

$$
S^{n-1} \stackrel{\tau}{\longrightarrow} \operatorname{BSpin}(n-1) \stackrel{i}{\longrightarrow} \operatorname{BSpin}(n) .
$$

Here $\tau$ classifies the tangent bundle of $S^{n-1}$, and $i$ is the standard inclusion.

For small values of $n$, these bundles have alternative descriptions as follows (for example see [18]).

For $n=2$ and up to isomorphism, the inclusion $\operatorname{Spin}(1) \backsim \operatorname{Spin}(2)$ is merely 
$\{ \pm 1\} \hookrightarrow S^{1}$, where $e^{\imath \theta} \in \operatorname{Spin}(2)$ acts on $\boldsymbol{R}^{2} \cong \boldsymbol{C}$ by $z \mapsto e^{2 i 0} z$. Hence 3.1 becomes

$$
S^{1} \stackrel{\tau}{\longrightarrow} R P^{\infty} \stackrel{i}{\longrightarrow} C P^{\infty},
$$

being the associated sphere bundle $S\left(\zeta^{2}\right)$ up to homotopy equivalence.

For $n=3$ and up to isomorphism, the inclusion $\operatorname{Spin}(2) \rightarrow \operatorname{Spin}(3)$ is the standard $S^{1} \hookrightarrow S^{3}$, and $\operatorname{Spin}(3)$ acts on $\boldsymbol{R}^{3}$ as conjugation on the pure quaternions. Hence 3.1 becomes

$$
S^{2} \stackrel{\tau}{\longrightarrow} C P^{\infty} \stackrel{i}{\longrightarrow} H P^{\infty},
$$

being the associated sphere bundle $S(\chi)$ up to homotopy equivalence. Moreover, pulling back $\chi$ to $B \operatorname{Sin}(2)$ yields the homotopy commutative diagram

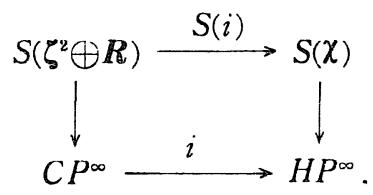

For $n=4$ and up to isomorphism, the inclusion $\operatorname{Spin}(3) \rightarrow \operatorname{Spin}(4)$ is the diagonal homomorphism $S^{3} \rightarrow S^{3} \times S^{3}$, where $(q, r) \subseteq \operatorname{Spin}(4)$ acts on $\boldsymbol{R}^{4} \cong \boldsymbol{H}$ by $v \mapsto q v r^{-1}$. Hence 3.1 becomes

$$
S^{3} \stackrel{\tau}{\longrightarrow} H P^{\infty} \stackrel{i}{\longrightarrow} H P^{\infty} \times H P^{\mathrm{c} \varepsilon} .
$$

Finally, for $n=5$ and up to isomorphism, the inclusion $\operatorname{Spin}(4) \rightarrow \operatorname{Spin}(5)$ is the embedding $S^{3} \times S^{3} \rightarrow S p(2)$ of diagonal matrices, where $S p i n(5)$ acts by conjugation on the real 5 -dimensional vector of $2 \times 2$ quaternionic Hermitian matrices of zero trace. Hence 3.1 becomes

$$
S^{4} \stackrel{\tau}{\longrightarrow} H P^{\infty} \times H P^{\infty} \stackrel{i}{\longrightarrow} B S p(2),
$$

where $\tau$ acts via the product of the homeomorphism $S^{4} \doteq H P^{1}$ with its homotopy inverse.

We may now take the mapping cone of each of these four projections $i$ by compactifying the corresponding vector bundle at $\infty$, so obtaining the following cofibre sequences

$$
\begin{gathered}
R P^{\infty} \stackrel{i}{\longrightarrow} C P^{\infty} \stackrel{f}{\longrightarrow} M\left(\zeta^{2}\right) \stackrel{b}{\longrightarrow} S R P^{\infty} \longrightarrow \cdots \\
C P^{\infty} \stackrel{i}{\longrightarrow} H P^{\infty} \stackrel{f}{\longrightarrow} M(\chi) \stackrel{b}{\longrightarrow} S C P^{\infty} \longrightarrow \cdots \\
H P^{\infty} \stackrel{i}{\longrightarrow} H P^{\infty} \times H P^{\infty} \stackrel{f}{\longrightarrow} M \operatorname{Spin}(4) \stackrel{b}{\longrightarrow} S H P^{\infty} \longrightarrow \cdots \\
H P^{\infty} \times H P^{\infty} \stackrel{i}{\longrightarrow} B S p(2) \stackrel{f}{\longrightarrow} \operatorname{MSpin}(5) \stackrel{b}{\longrightarrow} S\left(H P^{\infty} \times H P^{\infty}\right) \longrightarrow \cdots
\end{gathered}
$$


for the Thom complexes. Here each map $f$ is the inclusion of the zero section, and each map $b$ is its collapse; the context will always serve to distinguish between the cases. In addition, from 3.2, there is a useful homotopy commutative diagram

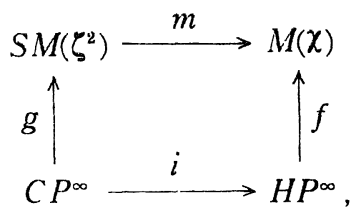

where $m=M(i)$ and $g$ is the inclusion of the zero section of $\zeta^{2} \oplus \boldsymbol{R}$.

In $[22]$ we proved that $\chi$ is $M S p$ orientable. We exhibited a virtual symplectic bundle $\varepsilon$ over

$$
S M(\chi) \doteq M(\chi \oplus \boldsymbol{R}) \doteq M\left(\xi \otimes_{H} \xi^{*}\right)
$$

which restricts to $\boldsymbol{\xi}-\boldsymbol{H}$ on the bottom cell $S^{4}$, and so defines an orientation class $t$ in $M S p^{3}(M(\mathcal{\chi}))$ by $s t=p f_{1}(\varepsilon)$. In turn, by virtue of 3.4, we may now record that $\zeta^{2}$ also admits an $M S p$ orientation class $u \in M S p^{2}\left(M\left(\zeta^{2}\right)\right)$, defined by $s u=m^{*} t$. Restricting to the zero sections then yields the symplectic Euler classes

$$
e=f^{*} t \in M S p^{3}\left(H P^{\infty}\right) \text { and } \quad d=f^{*} u \in M S p^{2}\left(C P^{\infty}\right)
$$

which play such a prominent role in the rest of our work.

In fact the class $d$ is a symplectic analogue of the 2 -series $[2] x \in M U^{2}\left(C P^{\infty}\right)$, defined as the pull-back under $f$ of the canonical complex orientation in $M U^{2}\left(M\left(\zeta^{2}\right)\right)$. Since this series is an important ingredient of the theory of $M U$ at the prime 2 , it is hardly surprising that we should find $d$ to be influential in the study of $M S p$.

We shall require an alternative description of the class $u$, implicit in the work of Toda and Kozima [25」. To explain this, we first remark that, by construction, we may identify the restriction of $M(\chi)$ over any skeleton $H P^{n}$ with the quasi projective space

$$
Q P^{n}=S_{+}^{4 n-1} \wedge S_{3} S^{3},
$$

where $S^{3}$ acts on the right of $S^{4 n-1}$ as usual, and on the left of $S^{3}$ as conjugation in the unit quaternions. This identification, which we shall use henceforth without comment, arises out of the obvious $S^{3}$-equivariant homeomorphism $h$ from the pure quaternions compactified as $\infty$ to $S^{3}$.

Now each $Q P^{n}$ admits a reflection map $j$ into $S p(n)$ (for example see [24]), and these maps are compatible at $n \rightarrow \infty$. We obtain

Lemma 3.6. The composition $M(\chi) \doteq Q P^{\infty} \stackrel{j}{\rightarrow} S p$ and the adjoint of $\varepsilon: S M(\chi)$ $\rightarrow B S p$ are homotopic. 
Proof. This is a straightforward exercise, carried out by showing that $\varepsilon$ and the adjoint of $j$ pull back to isomorphic symplectic bundles over the total space $S(\chi \oplus \boldsymbol{R})$.

Corollary 3.7. If $\Omega z \in M S p^{3}(S p)$ denotes the element created by looping the first Conner-Floyd symplectic Pontrjagin class, then $t=j^{*}(\Omega z)$ in $M S p^{3}(M(\chi))$.

Proof. This follows immediately from applying 3.6 to the definition of $t$ above.

We shall also need

Lemma 3.8. There is a homotopy commutative diagram

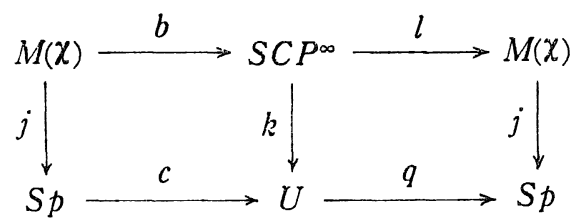

where $k$ is the complex reflection map and $l$ is the composition

defined from 3.4 .

$$
S C P^{\infty} \stackrel{s f}{\longrightarrow} S M\left(\zeta^{2}\right) \stackrel{m}{\longrightarrow} M(\chi)
$$

Proof. From [25], there is for each $n$ a homotopy commutative diagram

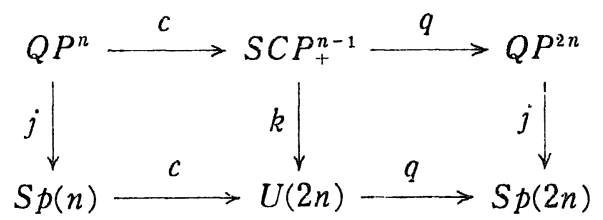

where the upper $c$ and $q$ are also standard complexification and symplectification maps. It thus suffices to prove that the two diagrams

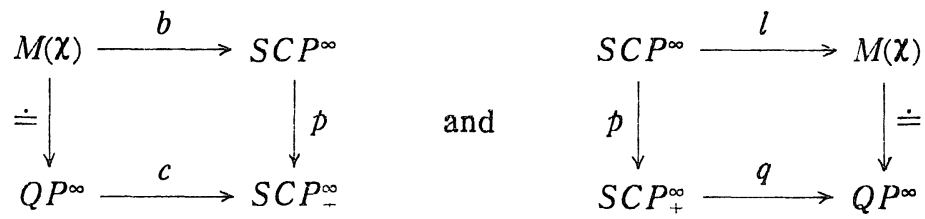

are, in turn, homotopy commutative, where $p$ identifies the two cone points of the suspension.

In fact a routine analysis, recalling the homeomorphism $h$ above, shows 
that both diagrams commute strictly.

Corollary 3.9. In $M S p^{3}(M(\chi))$, we have that $b^{*}(s d)=2 t$.

Proof. From 3.7 and 3.8 .

$$
\begin{aligned}
b^{*}(s d) & =b^{*}(s f)^{*} u=b^{*} l^{*} t \\
& =b^{*} l^{*} j^{*}(\Omega z)=j^{*}(q \cdot c)^{*}(\Omega z) .
\end{aligned}
$$

But $q \cdot c: S p \rightarrow S p$ is the map 2, which duplicates each symplectic matrix (for example see [1]) and has the property that $B 2$ classifies twice the universal symplectic bundle. Therefore

$$
(q \cdot c)^{*}(\Omega z)=2(\Omega z),
$$

and the proof is complete.

\section{§4. The Exact Sequences}

We now apply the symplectic cobordism functor to the first two of the cofibre sequences of 3.3 , and so obtain the long exact sequences

$$
\begin{gathered}
\cdots \stackrel{i^{*}}{\longrightarrow} M S p^{*-1}\left(R P^{\infty}\right) \stackrel{\delta}{\longrightarrow} M S p^{*}\left(M\left(\zeta^{2}\right)\right) \stackrel{f^{*}}{\longrightarrow} M S p^{*}\left(C P^{\infty}\right) \stackrel{i^{*}}{\longrightarrow} \cdots \\
\cdots \stackrel{i^{*}}{\longrightarrow} M S p^{*-1}\left(C P^{\infty}\right) \stackrel{\delta}{\longrightarrow} M S p^{*}(M(\chi)) \stackrel{f^{*}}{\longrightarrow} M S p^{*}\left(H P^{\infty}\right) \stackrel{i^{*}}{\longrightarrow} \cdots
\end{gathered}
$$

whose study is a primary aim of this section.

We have already observed that $\zeta^{2}$ and $\chi$ are $M S p$ orientable bundles, so we may immediately take advantage of the associated Thom isomorphisms to rewrite the above as

$$
\begin{aligned}
\ldots \stackrel{i^{*}}{\longrightarrow} M S p^{*-1}\left(R P^{\infty}\right) \stackrel{\delta}{\longrightarrow} M S p^{*-2}\left(C P_{+}^{\infty}\right) \stackrel{\cdot d}{\longrightarrow} M S p^{*}\left(C P^{\infty}\right) \stackrel{i^{*}}{\longrightarrow} \cdots \\
\cdots \stackrel{i^{*}}{\longrightarrow} M S p^{*-1}\left(C P^{\infty}\right) \stackrel{\delta}{\longrightarrow} M S p^{*-3}\left(H P_{+}^{\infty}\right) \stackrel{\cdot e}{\longrightarrow} M S p^{*}\left(H P^{\infty}\right) \stackrel{i^{*}}{\longrightarrow} \cdots
\end{aligned}
$$

where the maps $\cdot d$ and $\cdot e$ signify multiplication by the respective Euler classes introduced in 3.5. Before discussing the implications of these sequences, we must investigate $e$ in more detail.

Proposition 4.3. In $M S p^{3}\left(H P^{\infty}\right)$, we have that

$$
e=\sum_{i \geqq 1} \theta_{i} z^{i} \text {. }
$$

Proof. We recall that the class $u$ of 3.5 is constructed by analysing the 4-sphere bundle

$$
p: S\left(\boldsymbol{\chi} \oplus \boldsymbol{R}^{2}\right) \longrightarrow H P^{\infty}
$$


whose total space admits symplectic line bundles $\nu$ and $p^{*} \xi$. There is a commutative diagram of cofibre sequences

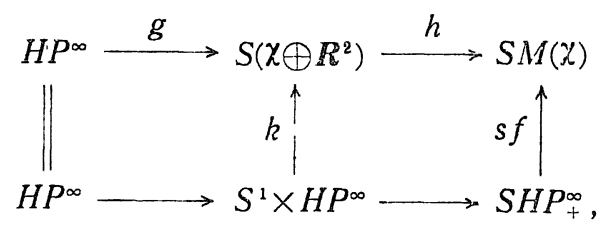

where $g$ is the section described by the point $(0,1) \in \boldsymbol{R}^{2}$, and $k$ is its extension over the unit circle. Following [22] (wherein $\nu$ is labelled $\zeta$ ), the symplectic bundle $\varepsilon$ over $S M(\chi)$ satisfies $h^{*} \varepsilon=\nu-p^{*} \xi$, whilst it follows from the definitions that $k^{*} \nu=\eta \otimes_{R} \xi$. Incorporating this information into the diagram yields

$$
\begin{aligned}
s e & =(s f)^{*} u=k^{*} h^{*} u=k^{*} h^{*} p f_{1}(\varepsilon) \\
& =k^{*} p f_{1}\left(\nu-p^{*} \xi\right)=p f_{1}\left(\eta \otimes_{R} \xi-\xi\right) \\
& =s\left(\sum_{i \geqq 1} \theta_{i} z^{i}\right) .
\end{aligned}
$$

Desuspending then yields the result.

We now have a better hold on the homomorphism $e$. In particular, we need to refer to its kernel Ker $e$ and cokernel Cok $e$, both of which are, of course, $M S p_{*}$ modules. By virtue of 4.3 , we have the descriptions

$$
\operatorname{Ker} e=\left\{\sum_{j \geqq 1} \alpha_{j} z^{j}: \sum_{i+j=k} \theta_{i} \alpha_{j}=0 \quad \forall k\right\}
$$

and Cok $e=M S p^{*}[[z]] /(e)$, where $(e)$ denotes the ideal generated by $e$.

Describing the homomorphism $\cdot d$ is more difficult, since it acts on $M S p^{*}\left(C P^{\infty}\right)$, which is not free over $M S p^{*}$; however, as we shall see, a few properties can be gleaned from various of our formulae.

We now state our structure theorem.

Theorem 4.4. There are short exact sequences of MSp* modules

$$
\begin{aligned}
& 0 \longrightarrow \operatorname{Cok} e \stackrel{i^{*}}{\longrightarrow} M S p^{*}\left(C P^{\infty}\right) \stackrel{\delta}{\longrightarrow} \operatorname{Ker} e \longrightarrow 0 \\
& 0 \longrightarrow \operatorname{Cok} d \stackrel{i^{*}}{\longrightarrow} M S p^{*}\left(R P^{\infty}\right) \stackrel{\delta}{\longrightarrow} \operatorname{Ker} d \longrightarrow 0 .
\end{aligned}
$$

Proof. These follow at once by restriction to the relevant parts of 4.2 .

Note that 3.9 tells us that $d$ maps non-trivially to Ker $e$ in the first of these sequences.

It is a straightforward matter to dualise 4.4 so as to refer to the bordism modules instead. The only significant modification is to interpret the homo- 
morphisms $d$ and $e$ in terms of cap products, which therefore lower, rather than raise, dimension.

To proceed further, we must now examine the last of the cofibre sequences of 3.3 , whose long exact sequence may be displayed as

$$
\ldots \stackrel{i^{*}}{\longrightarrow} M S p^{*-1}[[y, z]] \stackrel{\delta}{\longrightarrow} M S p^{*}(M S p i n(5)) \stackrel{f^{*}}{\longrightarrow} M S p^{*}(B S p(2)) \stackrel{i^{*}}{\longrightarrow} \ldots
$$

by taking due note of the Künneth isomorphism 2.1, and writing $y$ for the generator of the symplectic cobordism of the first copy of $H P^{\infty}$. This sequence allows us to deduce the following.

Proposition 4.6. Every Spin(5) bundle is MSp orientable, and has zero symplectic Euler class.

Proof. We first show that the element $v=\delta y$ in $M S p^{5}(M \operatorname{Spin}(5))$ restricts to a generator on the bottom cell, thereby establishing that it is an orientation class. To do this, it suffices to consider the pull back of $y$ along the inclusion of the fibre $S^{4} \rightarrow H P^{\infty} \times H P^{\infty}$. This map $\tau$ is described explicitly in $\S 3$, from which we deduce that $\tau^{*} y$ is indeed a generator of $M S p^{4}\left(S^{4}\right)$, as required.

Given this choice of orientation class, the symplectic Euler class $i * \delta y$ is clearly zero. But any other orientation class agrees with $v$ up to multiplication by a unit of $M S p^{0}\left(B S p(2)_{+}\right)$, whence the result follows.

This simple fact has striking consequences.

Proposition 4.7. In $M S p^{5}\left(C P^{\infty} \wedge H P^{\infty}\right)$, we have that $d \otimes e=0$.

Proof. The bundle $\zeta^{2} \times \chi$ over $C P^{\infty} \times H P^{\infty}$ is the universal $\operatorname{Spin}(2) \times \operatorname{Spin}(3)$ bundle, with orientation class $u \otimes t$ in $M S p^{5}\left(M\left(\zeta^{2}\right) \wedge M(\chi)\right)$ : so our equation follows by applying 4.6 .

Corollary 4.8. In $M S p^{*}\left(C P^{\infty}\right)$, the relation $\theta_{i} d=0$ holds for all $i>0$.

Proof. This results from combining 4.3 with 4.7 .

The above relation allows us to make an additional comment concerning the structure theorem 4.4, namely that Ker $d$ contains the ideal

$$
\left(\phi_{1}, \phi_{2}, \cdots, \phi_{i}, \cdots\right) \subset M S p^{*}\left(C P_{+}^{\infty}\right)
$$

generated by the elements $\phi_{i}$. 


\section{§. The Rôle of the Real Projective Plane}

We now investigate the commutative ladder of cofibre sequences

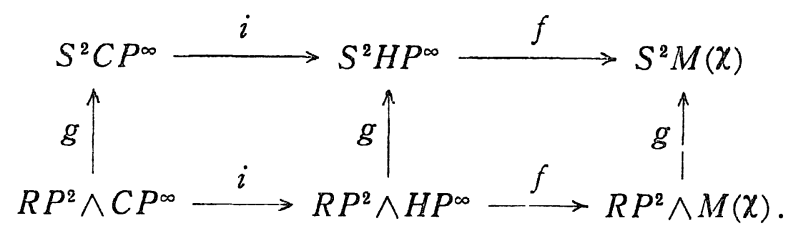

Here the vertical maps are derived from the projection $g: R P^{2} \rightarrow S^{2}$ of $\S 2$ by smashing with the identity on the relevant projective space, whilst the horizontal maps are obtained from $i$ and $f$ of 3.3 by smashing with the identity on $S^{2}$ and $R P^{2}$ respectively; although an abuse of notation, such a convention simplifies several formulae below. Introducing $R P^{2}$ in this fashion will allow us, in the two main results of this section, to make geometrical constructions with crucial algebraic consequences.

We shall concentrate on the induced ladder

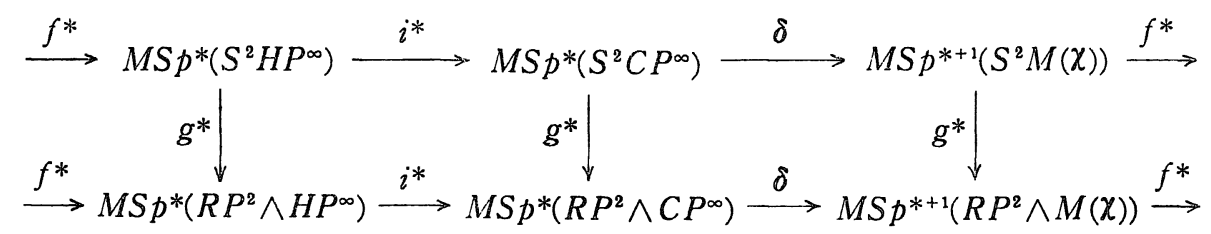

of long exact sequences. Remark that, since $M S p^{*}(M(\chi))$ is a free $M S p^{*}$ module by virtue of the Thom isomorphism, there is a Künneth isomorphism

$$
M S p^{*}\left(R P^{2} \wedge M(\chi)\right) \cong M S p^{*}\left(R P^{2}\right) \otimes M S p^{*}(M(\chi)) .
$$

Now recall from 2.4 that $r(2)$ denotes the pull back $g^{*} s(2)$ in $M S p^{2}\left(R P^{2}\right)$.

Proposition 5.1. In $M S p^{7}\left(R P^{2} \wedge M(\chi)\right)$ we have that

$$
\delta\left(r(2) \otimes d^{2}\right)=\eta r(2) \otimes u^{2} .
$$

Proof. We first show that in the lower long exact sequence, $r(2) \otimes d$ lies in the image of $i^{*}$.

Consider the virtual $S p$ bundle $(\eta-\boldsymbol{R}) \otimes_{\boldsymbol{R}}(\boldsymbol{\xi}-\boldsymbol{H})$ over $R P^{2} \wedge H P^{\infty}$; it pulls back over $R P^{2} \wedge C P^{\infty}$ to

$$
\begin{aligned}
(\eta-\boldsymbol{R}) \otimes_{R}(q \boldsymbol{\zeta}-\boldsymbol{H}) & =(c \eta-\boldsymbol{C}) \otimes_{c}(\boldsymbol{\zeta} \oplus \bar{\zeta}-\boldsymbol{H}) \\
& =\left((c \eta-\boldsymbol{C}) \otimes_{c}(\boldsymbol{\zeta}-\boldsymbol{C})\right) \oplus\left((\overline{c \eta}-\boldsymbol{C}) \otimes_{c}(\bar{\zeta}-\boldsymbol{C})\right),
\end{aligned}
$$

bearing in mind that $c \eta=\overline{c \eta}$ over $R P^{\infty}$. By standard bundle computations (for example see [13] again), $c \eta=g^{*}{ }^{*}$ over $R P^{2}$, whilst over $S^{2} C P^{\infty}$ 


$$
\begin{aligned}
\left((\boldsymbol{\zeta}-\boldsymbol{C}) \otimes_{\boldsymbol{c}}(\boldsymbol{\zeta}-\boldsymbol{C})\right) \oplus\left((\bar{\zeta}-\boldsymbol{C}) \otimes_{\boldsymbol{c}}(\bar{\zeta}-\boldsymbol{C})\right) & =q(\boldsymbol{\zeta}-\boldsymbol{C}) \otimes_{c}(\boldsymbol{\zeta}-\boldsymbol{C}) \\
& =(s l)^{*} \boldsymbol{\varepsilon}
\end{aligned}
$$

from 3.6 and 3.8. Combining these facts gives

$$
g^{*}(s l)^{*} \varepsilon=i *\left((\eta-\boldsymbol{R}) \otimes_{\boldsymbol{R}}(\boldsymbol{\xi}-\boldsymbol{H})\right),
$$

and taking $p f_{1}()$ of both sides yields

$$
r(2) \bigotimes d=i^{*}\left(\sum_{i \geqq 1} \theta_{i}(2) z^{2}\right)
$$

as sought.

Our proof is completed by recalling (for example from [9]) that $\delta$ is a $M S p^{*}\left(R P^{2} \wedge H P^{\infty}\right)$ module map. Thus

$$
\begin{aligned}
\delta\left(r(2) \otimes d^{2}\right)=\delta(r(2) \otimes d \cdot d) & =\delta\left(i *\left(\sum_{i \geqq 1} \theta_{i}(2) z^{2}\right) \cdot d\right) \quad \text { from above } \\
& =\left(\sum_{i \geqq 1} \theta_{i}(2) z^{i}\right) \cdot \delta d \\
& =\left(\sum_{i \geqq 1} \theta_{i}(2) z^{2}\right) \cdot 2 u \quad \text { from } 3.9 \\
& =\eta\left(\sum_{i \geqq 1} \theta_{i} r(2) z^{i}\right) \cdot u \quad \text { from } 2.4 \\
& =\eta r(2) \otimes e \cdot u \quad \text { from } 4.3 .
\end{aligned}
$$

But $e \cdot u=u^{2}$ by definition of the Euler class, and so we obtain our desired formula.

Corollary 5.2. In $M S p^{5}(M(\chi))$, we have that $\delta d^{2} \equiv \eta u^{2} \bmod 2$.

Proof. Since $g^{*}: M S p^{*}\left(S^{2}\right) \rightarrow M S p^{*}\left(R P^{2}\right)$ has kernel $(2 s(2))$, the result follows directly from the proposition.

A valuable alternative perspective is offered by the transfer map $p_{\|}$associated to the $S^{2}$-bundle projection $p: C P^{2 n+1} \rightarrow H P^{n}$, where $n$ is arbitrary [4]. In this context, 5.2 tells us that $p_{\sharp} d \equiv \eta e \bmod 2$ in $M S p^{2}\left(H P^{n}\right)$, where $d$ and $e$ are suitably restricted to the finite skeleta. Similarly, 3.9 may be restated as $p_{\eta 1} 1=2$ in $M S p^{0}\left(H P_{+}^{\infty}\right)$. These formulae are discussed in [11].

Our second task in this section is to introduce new elements $\phi_{i}(n)$ in $M S p^{8-4 i}\left(C P^{n}\right)$. These are defined in the same spirit as Buhštaber's elements $\theta_{i}(n)$, by means of the equation

$$
p f_{2}\left(\left(r \zeta-\boldsymbol{R}^{2}\right) \bigotimes_{\boldsymbol{R}}(\xi-\boldsymbol{H})\right)=\sum_{i \geqq 1} \psi_{i}(n) z^{2}
$$

in $M S p^{8}\left(C P^{n} \wedge H P^{\infty}\right)$. Clearly, given any $m<n$, each $\phi_{i}(n)$ restricts to $\phi_{i}(m)$ under the inclusion $C P^{m} \subset C P^{n}$. 
For $n=1$, we may use the suspension isomorphism to rewrite $\phi_{i}(1)$ in $M S p^{8-4 i}\left(C P^{1}\right)$ as an element in $M S p_{4 i-6}$, which we shall label $\phi_{i}$; its identification is of special interest, and again involves considering $R P^{2}$.

Proposition 5.4. For all $i \geqq 1$, we have that

$$
g^{*} \phi_{4 i}(1)=\phi_{i}{ }^{2} r(2), \quad g^{*} \phi_{4 i+2}(1)=0, \quad \text { and } \quad g^{*} \phi_{2 i+1}(1)=\eta \phi_{i} r(2)
$$

in $M S p^{*}\left(R P^{2}\right)$.

Proof. Since $g *(r \zeta)=2 \eta$ over $R P^{2}$ (as already noted in 2.4 ), it follows that

$$
g^{*}\left(\left(r \boldsymbol{\zeta}-\boldsymbol{R}^{2}\right) \otimes_{R}(\xi-\boldsymbol{H})\right)=2(\eta-\boldsymbol{R}) \otimes_{R}(\xi-\boldsymbol{H})
$$

over $R P^{2} \wedge H P^{\infty}$.

Now the total symplectic Pontryagin class $p f\left((\eta-R) \otimes_{R}(\xi-H)\right)$ is given by

$$
\begin{aligned}
p f\left(\eta \otimes_{R} \xi\right) \cdot p f(\xi)^{-1} & =\left(1+z+\sum_{i \geqq 1} \theta_{i}(2) z^{i}\right)(1+z)^{-1} \\
& =1+\sum_{j \geqq 0} \sum_{i \geqq 1}(-1)^{j} \theta_{i}(2) z^{i+j} .
\end{aligned}
$$

Hence applying $p f_{2}()$ to 5.5 and making use of 2.4 and 2.5 yields

$$
\begin{aligned}
g^{*} \sum_{i \geqq 1} \phi_{i}(1) z^{i} & =\left(\sum_{i \geqq 1} \theta_{i}(2) z^{i}\right)^{2}+2 \sum_{i \geqq 1} \theta_{i}(2) z^{i+1} \\
& =\sum_{i \geqq 1} \theta_{i}{ }^{2} r(2) z^{2 i}+\eta \sum_{i \geqq 1} \theta_{i} r(2) z^{i+1} .
\end{aligned}
$$

Equating coefficients and appealing to Roush's theorem establishes our formulae.

Corollary 5.6. For all $i \geqq 1$, we have that in $M S p_{*}$.

$$
\phi_{4 i} \equiv \phi_{i}{ }^{2} \bmod 2, \quad \phi_{4 i+2} \equiv 0 \bmod 2, \quad \text { and } \phi_{2 i+1} \equiv \eta \phi_{i} \bmod 2
$$

Proof. These follows by direct analogy with the proof of 5.2 .

\section{§ 6. Applications to $M S p_{\text {* }}$}

We are now in a position to apply the results of $\S 5$ and exhibit our relations amongst the elements $\phi_{i}$.

Theorem 6.1. The relation

$$
\eta \theta_{i} \theta_{\jmath}=0
$$

holds in $M S p_{4(i+\jmath)-5}$, for all $i, j \geqq 1$. 
Proof. Combining 4.2 and 5.2, we deduce that $\delta: M S p^{*}\left(C P^{\infty}\right) \rightarrow M S p^{*-2}\left(H P_{+}^{\infty}\right)$, the homomorphism induced by the map $b: M(\chi) \rightarrow S C P^{\infty}$, satisfies $\delta d^{2}=\eta e \bmod 2$. Therefore, after smashing with the identity on $H P^{\infty}$, the composition

$$
\delta: M S p^{*}\left(C P^{\infty} \wedge H P_{+}^{\infty}\right) \longrightarrow M S p^{*-3}\left(H P_{+}^{\infty} \wedge H P_{+}^{\infty}\right) \cong M S p^{*-3}[[y, z]]
$$

acts such that $\delta\left(d^{2} \otimes e\right)=\eta e \otimes e$. But $d \otimes e$ is zero by 4.7 , whence $\eta e \otimes e$ in zero also.

Since $\theta_{i} \theta_{j}$ is the coefficient of $y^{\prime} z^{j}$ in $e \otimes e$ by 4.3 , the result follows.

Corollary 6.2. Any symplectic Euler class of a $\operatorname{Spin}(3) \times \operatorname{Spin}(3)$ bundle is annihilated by $\eta$.

Proof. The universal example is $e \otimes e$, which is shown to be so annihilated in the proof of the proposition.

It is worth noting at this point that, in contrast to the lower dimensional cases, the universal $\operatorname{Spin}(6)$ bundle is not $M S p$ orientable. As pointed out to us by A. Baker, the Stiefel-Whitney class $w_{6}$ provides an obstruction.

We conclude with our nilpotence formula.

Theorem 6.3. The relation

$$
\phi_{i}{ }^{2 \jmath+2} \phi_{\jmath}=0
$$

holds in $M S p_{*}$ for any $i \geqq j \geqq 1$.

Proof. Using 5.6, we see that in 4.2

$$
\delta \phi_{1 i}(\infty) \equiv \phi_{\imath}{ }^{2}+\sum_{k \leq 1} \mu_{i, k} z^{k} \bmod 2
$$

for certain elements $\mu_{2, k}$ in $M S p_{16 \imath+4 k-6}$. Hence

$$
\left(\phi_{i}{ }^{2}+\sum_{k \geqq 1} \mu_{\imath, k} z^{k}\right)\left(\eta z+\sum_{j \geqq 1} \phi_{J} z^{2 \jmath}\right)=0
$$

in $M S p^{9-16 \imath}\left(H P^{\circ}\right)$. Setting coefficients to zero yields the system of $i+1$ equations

$$
\begin{aligned}
\eta \phi_{i}{ }^{2} & =0 \\
& \vdots \\
\phi_{J} \phi_{i}{ }^{2} & =\sum_{k=1}^{j-1} \phi_{J-k} \mu_{2,2 k}+\eta \mu_{i, 2 J-1} \\
& \vdots \\
\phi_{i}{ }^{3} & =\sum_{k=1}^{i-1} \phi_{i-k} \mu_{i, 2 k}+\eta \mu_{i, 2 i-1}
\end{aligned}
$$

in $M S p_{*}$.

To deduce our formula, we now have only to multiply the $(j+1)$ th of these 
by $\phi_{i}{ }^{2 j}$, for every $i \geqq j \geqq 1$, and substitute in each equation from the previous ones.

Corollary 6.4 . The relation

$$
\phi_{i}{ }^{2 \imath+3}=0
$$

holds in $M S p_{(2 i+3)(8 i-3)}$ for any $i \geqq 1$.

\section{References}

[1] Adams, J.F., Lectures on Lic groups, W. A. Benjamin, New York, 1969.

[2] Atiyah, M.F., Bott, R. and Shapiro, A., Clifford modules, Topology, 3 (1964), 338.

[3] Bakuradze, M.R. and Nadiradze, R.G., Cohomological realization of two-valued formal groups and their applications, Bull. Acad. Sci. Georgian SSR, 28 (1987), 21-24.

[4] Becker, J.C. and Gottlieb, D.H., The transfer map and fiber bundles, Topology, 14 (1975), 1-12.

[5] Botvinnik, B.I., Manifolds with singularitics and geometric realization of the Adams-Novikov spectral sequence, to appear, LMS Lect. Note Ser.

[6] Buhštaber, V.M., Characteristic classes in cobordism and topological applications of theories of one- and two-valued formal groups, Itogi Nauki $i$ Tekhniki, 10 (1978), $5-178$.

[7] Cartan, H., Périodicité des groupes d'homotopie stables des groupes classiques, d'apres Bott, Sem. Henri Cartan (1959/60).

[8] Conner, P.E. and Floyd, E.E., The relation of cobordism to $K$ theories, Lect. Notes in Math. 28, Springer-Verlag, 1966.

[9] Dyer, E., Cohomology theories, W. A. Benjamin, New York, 1969.

[10] Gorbunov, V.G. and Vershinin, V. V., Ray's elements as obstructions to orientability of symplectic cobordism, Sov. Math. Dokl., 32 (1985), 855-858.

[11] Gorbunov, V.G., The symplectic cobordism of projective spaces, Mat. Sb., 3 (1989), 345-357.

[12] Hopkins, M. J., Global methods in homotopy theory, in Homotopy theory, ed. E. Rees and J.D.S. Jones, LMS Lect. Note Ser., 117 (1987), 73-96.

[13] Husemoller, D., Fibre Bundles, McGraw-Hill, New York, 1966.

[14] Kochman, S.O., The symplectic cobordism ring I, Mem. Amer. Math. Soc., 228 (1980).

[15] - The symplectic cobordism ring II, Mem. Amer. Math. Soc., 271 (1982).

[16] Landweber, P.S., Elliptic cohomology and modular forms, Lect. Notes in Math. 1326, Springer-Verlag 1988, 55-68.

[17] Morisugi, K., Massey products in $M S p_{*}$ and its applications, Jour. Math. of Kyoto Univ. 23-2 (1983), 239-263.

[18] Porteous, I.R., Topological geometry, Van Norstrand Reinhold, London, 1969.

[19] Ravenel, D.C., Complex cobordism and stable homotopy groups of spheres, Academic Press, Orlando, 1986.

[20] Ray, N., Indecomposables in Tors MSp $p_{*}$, Topology, 10 (1971), 261-270.

[21] - Symbolic calculus: a 19th century approach to $M U$ and $B P$, in Homotopy theory, ed. E. Rees and J.D.S. Jones, LMS Lect. Note Ser., 117 (1987), 195-238.

[22] Ray, N. and Switzer, R., The algebraic topology of Alexander's manifolds, Mem. Amer. Math. Soc., 193 (1977), 28-42. 
[23] Roush, F.W., On some torsion classes in symplectic bordism, unpublished.

[24] Steenrod, N.E. and Epstein, D.B.A., Cohomology operations, Annals of Math. Studies, 50, Princeton University Press, 1962.

[25] Toda, H. and Kozima, K., The symplectic Lazard ring, J. Math. Kyoto Univ., 22 (1982), 131-153.

[26] Vershinin, V.V., Symplectic cobordism with singularities, Math, USSR Izvestiya, 22 (1984), 211-226.

[27] — Computation of the symplectic cobordism ring in dimensions less than 32 , and the non-triviality of the majority of triple products of Ray's elements, Sibirsk Mat. Zh., 24 (1983), 50-63. 
